\title{
Electron Accumulation Induces Efficiency Bottleneck for Hydrogen Production in Carbon Nitride Photocatalysts
}

\author{
Wenxing Yang ${ }^{1 \dagger}$, Robert Godin ${ }^{1 \ddagger}$, Hatice Kasap², Benjamin Moss ${ }^{1}$, Yifan Dong ${ }^{1}$, Sam A. J. Hillman ${ }^{1}$, Ludmilla \\ Steier $^{1}$, Erwin Reisner ${ }^{2}$, James R. Durrant ${ }^{1 *}$ \\ ${ }^{1}$ Department of Chemistry and Centre for Plastic Electronics, Imperial College London, South Kensington Campus, \\ London SW7 2AZ, U.K. \\ ${ }^{2}$ Department of Chemistry, University of Cambridge, Lensfield Road, Cambridge CB2 1EW, U.K. \\ *Email: j.durrant@imperial.ac.uk \\ ${ }^{\dagger}$ Current address:
}

Department of Chemistry, Emory University, Atlanta, Georgia 30322, United States

${ }^{\ddagger}$ Current address:

Department of Chemistry, The University of British Columbia, Okanagan Campus, 3247 University Way, Kelowna, British Columbia V1V 1V7, Canada

\begin{abstract}
This study addresses the light intensity dependence of charge accumulation in a photocatalyst suspension, and its impact on both charge recombination kinetics and steady-state $\mathrm{H}_{2}$ evolution efficiency. Cyanamide surface functionalized melon-type carbon nitride $\left({ }^{\mathrm{NCN}} \mathrm{CN}_{\mathrm{x}}\right.$ ) has been selected as an example of emerging carbon nitrides photocatalysts because of its excellent charge storage ability. Transient spectroscopic studies (from ps to s) show that the bimolecular recombination of photogenerated electrons and holes in ${ }^{\mathrm{NCN}} \mathrm{CN}_{\mathrm{x}}$ can be well described by a random walk model. Remarkably, the addition of hole scavengers such as 4-methylbenzyl alcohol can lead to 400-fold faster recombination kinetics (lifetime shortening to $\sim 10 \mathrm{ps}$ ). We show that this acceleration is not the direct result of ultrafast hole extraction by the scavenger, but is rather caused by long-lived electron accumulation in ${ }^{\mathrm{NCN}} \mathrm{CN}_{\mathrm{x}}$ after hole extraction. The dispersive pseudo-first order recombination kinetics become controlled by the density of accumulated electrons. $\mathrm{H}_{2}$ production and steady-state spectroscopic measurements indicate that the accelerated recombination caused by electron accumulation limits the $\mathrm{H}_{2}$ generation efficiency. The addition of a reversible electron acceptor and mediator, methyl viologen $\left(\mathrm{MV}^{2+}\right)$, accelerates the extraction of electrons from the ${ }^{{ }^{N C N}} \mathrm{CN}_{\mathrm{x}}$ and increases the $\mathrm{H}_{2}$ production efficiency under one sun irradiation by more than $30 \%$. These results demonstrate quantitatively that whilst long-lived electrons are essential to drive photoinduced $\mathrm{H}_{2}$ generation in many photocatalysts, excessive electron accumulation may result in accelerated recombination losses and lower performance, and thus highlight the importance of efficient electron and hole extraction in enabling efficient water splitting photocatalysts.
\end{abstract}




\section{Introduction}

Artificial photosynthesis, a process employing sunlight to drive the generation of fuels from water and $\mathrm{CO}_{2}$, is attracting extensive research interest due to its potential for developing sustainable and carbon neutral alternatives to fossil fuels. ${ }^{1-}$

${ }^{3}$ Its operational principle involves photon absorption, charge separation and transfer of photogenerated electrons and holes to either electrochemical interfaces to drive surface chemistry (e.g., the formation of the $\mathrm{H}-\mathrm{H}$ bond from proton reduction) and/or an external electrical circuit. In artificial photosynthetic systems, many of the processes determining device efficiency are non-linear in either light or charge carrier density. These phenomena include the intrinsic photophysical processes of the light absorber. For example, bimolecular recombination processes ${ }^{4,5}$ and multiexciton interaction ${ }^{6,7}$ have been shown to be strongly nonlinear with respect to the carrier densities in metal oxide electrodes ${ }^{4,5}$ and quantum dots. ${ }^{6,7}$ In addition, photocatalytic systems often exhibit large asymmetries in the kinetics of charge transfer and extraction, dependent upon the nature of the extraction processes. Recently, we have recently reported that for water oxidation on metal oxide photoanode, the reaction order of hole extraction can change from 1 to 3 with the increase of the surface carrier density. ${ }^{8,9}$ As such, the photophysics and performance of these systems can be strongly dependent upon irradiation light intensity. ${ }^{4,10}$ These non-linearities and asymmetries in kinetics are not only of fundamental importance to understanding device efficiency but relevant to practical device application under the broad range of light intensities supplied by solar irradiation. Therefore, understanding the impact of such non-linear processes upon photocatalytic efficiencies is a key, but relatively unexplored, challenge and can provide vital insights for the development of efficient artificial photosynthetic systems. ${ }^{11}$

In studies of photocatalyst suspensions, hole (or electron) scavengers are often added to remove one charge carrier efficiently from the light absorber, in order to facilitate studies of the complimentary oxidation/reduction reaction (e.g., water oxidation to $\mathrm{O}_{2}$ /proton reduction to $\mathrm{H}_{2}$ ). This scavenging process can, however, create a strong kinetic asymmetry in the carrier extraction from the light absorber, resulting in the accumulation of one type of charge, as detailed illustrated in Scheme S1. Surprisingly, while several studies have indicated large kinetic differences in the electron and hole transfer processes, ${ }^{12,13}$ the impact of such asymmetries on charge accumulation and their correlation to device performance has been rarely studied. ${ }^{13,14}$ Such studies are important both for the technological function of devices under variable solar irradiation and for the correct interpretation of the pulsed laser spectroscopic measurements, where the irradiation differs substantially in temporal profile and intensity from solar irradiation. Herein, we focus on the study of the light intensity dependent asymmetric charge accumulation for a particularly promising photocatalytic material, carbon nitride $\left(\mathrm{CN}_{\mathrm{x}}\right)$ (also referred to in the literature as graphitic carbon nitride, $\mathrm{g}-\mathrm{C}_{3} \mathrm{~N}_{4}$ ).

$\mathrm{CN}_{\mathrm{x}}$ is an important class of organic photocatalysts, ${ }^{15-17}$ which has attracted particular research interest since its first demonstration of photocatalytic $\mathrm{H}_{2}$ production in $2009 .{ }^{18}$ Further research has been motivated by their tunable bandgap, low cost of synthesis and ease of surface functionalization. By simply thermally treating melon ${ }^{19}$, urea ${ }^{20}$ and other carbon-nitrogen precursors, a variety of $\mathrm{CN}_{\mathrm{x}}$ photocatalysts can be easily synthesized typically with a $2 \mathrm{D}$ graphitic structure, ${ }^{21}$ or as polymeric ribbons connected by $\mathrm{H}$ bonds. ${ }^{22}$ Its suitable band edge positions, strong light absorption, and high stability have facilitated $\mathrm{CN}_{\mathrm{x}}$ to achieve wide application for both $\mathrm{CO}_{2}$ reduction ${ }^{23,24}$ and $\mathrm{H}_{2}$ production ${ }^{25}$ in the presence of sacrificial donors. However, very few detailed photophysical studies of $\mathrm{CN}_{\mathrm{x}}$ have been reported to date, ${ }^{12,26-}$ ${ }^{30}$ limiting our understanding of the carrier dynamics determining its photocatalytic performance. This is partially due to its tendency to aggregate in solution and therefore form optically scattering suspensions, which are more challenging to study spectroscopically. ${ }^{29}$ Elucidation of the carrier dynamics in the $\mathrm{CN}_{\mathrm{x}}$ and their correlation with efficiency is therefore needed to enable further development of this promising photocatalytic system. ${ }^{25}$

In this study, we focus upon post-synthetically modified (KSCN treated) melamine-derived carbon nitride, referred to herein as ${ }^{\mathrm{NCN}} \mathrm{CN}_{\mathrm{x}}$. The introduction of cyanamide $(\mathrm{NCN})$ terminal groups has been previously shown to significantly improve $\mathrm{H}_{2}$ production efficiency, ${ }^{31}$ and to achieve rapid hole extraction by 4-methylbenzyl alcohol (4-MBA) to produce selectively the desired product benzyl aldehyde with high conversion yields. ${ }^{32}{ }^{\mathrm{NCN}} \mathrm{CN}_{\mathrm{x}}$ suspensions were also demonstrated to accumulate electrons for several hours during irradiation, making them appealing for photocatalysis using light/dark irradiation and solar battery applications. ${ }^{33,34}$ Moreover, unlike in urea-derived $\mathrm{CN}_{\mathrm{x}},{ }^{12}$ these long-lived, accumulated electrons retain sufficient driving force to reduce protons following discharging in the dark with a suitable co-catalyst. The long-lived electrons generated in this system offer an ideal opportunity to study the impact of electron accumulation upon carrier kinetics and $\mathrm{H}_{2}$ production as a model photocatalytic system.

Herein, we conduct photophysical studies on ${ }^{\mathrm{NCN}} \mathrm{CN}_{\mathrm{x}}$ suspensions on timescales ranging from ps to $\mathrm{s}$ with two aims: (i) to contribute to the current understanding of photophysical properties of this particular $\mathrm{CN}_{\mathrm{x}}$ material and (ii) to utilize 
the strong electron accumulation observed in ${ }^{{ }^{N C N}} \mathrm{CN}_{\mathrm{x}}$ to investigate the impact of charge accumulation on carrier dynamics and its correlation with photocatalytic performance. We start by detailing a procedure to produce a welldispersed suspension of this system, enabling detailed spectroscopic studies, and discuss its basic material and optical properties. We subsequently study the photophysical processes for the stable ${ }^{\mathrm{NCN}} \mathrm{CN}_{\mathrm{x}}$ suspension and then focus on the impact of electron accumulation within this ${ }^{\mathrm{NCN}} \mathrm{CN}_{\mathrm{x}}$ induced by irradiation in the presence of 4-MBA and discuss the resultant acceleration of recombination kinetics under these conditions. These kinetic studies are further correlated with the $\mathrm{H}_{2}$ production efficiency of the system under various steady-state illumination intensity. Finally, we explore the usage of a redox mediator to mitigate the negative impact of electron accumulation on the photocatalytic $\mathrm{H}_{2}$ production. These results provide insights regarding the photophysical properties of the $\mathrm{CN}_{\mathrm{x}}$ materials and, more importantly, demonstrate that the efficiency bottleneck of such photocatalysts could result from the accelerated recombination caused by charge accumulation.

\section{Result and Discussion.}

\section{Steady-State Characterization}

(a)

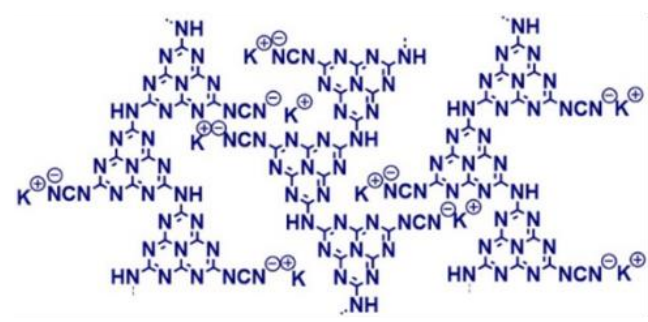

(c)

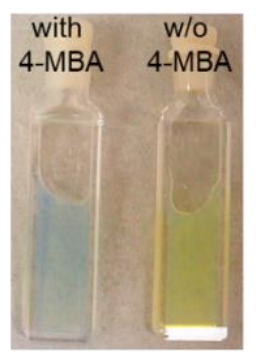

(b)

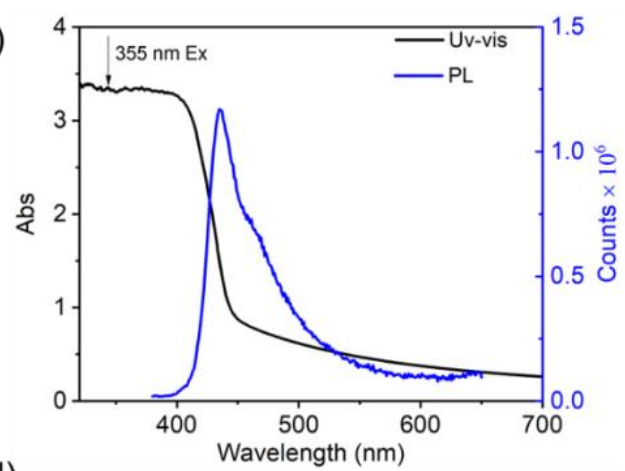

(d)

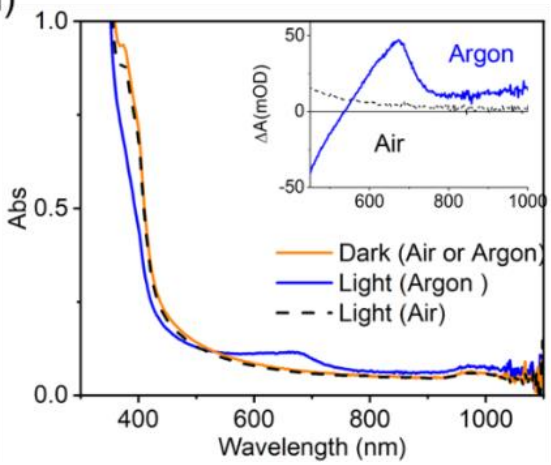

Figure 1. Basic properties of the ${ }^{\mathrm{NCN}} \mathrm{CN}_{\mathrm{x}}$ photocatalyst used in the present study. (a) Chemical structure. (b) UVvis and PL spectra of ${ }^{{ }^{N C N}}{ }^{C N} N_{x}$ dispersed in water $(1 \mathrm{mg} / \mathrm{ml})$. (c) The visible change of the sample in the electron accumulated state (blue, left) as compared to the original state (yellow, right) caused by hole extraction by 4-MBA. (d) Absorption spectra of ${ }^{\mathrm{NCN}} \mathrm{CN}_{\mathrm{x}}$ samples before and after illumination $(\sim 10 \mathrm{~min})$ in argon and air in the presence of 4MBA. Yellow line: the spectra before illumination; Blue line: after illumination under argon; Black dash line: after illumination under air. Inset: the corresponding absorption difference spectra due to light illumination.

Figure 1a shows the chemical structure of the ${ }^{\mathrm{NCN}} \mathrm{CN}_{\mathrm{x}}$ photocatalyst used in the present study, which was synthesized at $550{ }^{\circ} \mathrm{C}$ using melamine as the precursor, followed by a KSCN post-synthetic treatment as reported previously. ${ }^{31,32}$ To produce a stable and well-dispersed solution for optical measurements, the ${ }^{{ }^{N C N}} \mathrm{CN}_{\mathrm{x}}$ suspension $(3 \mathrm{mg} / \mathrm{ml})$ was firstly sonicated in water overnight to disperse the particles and then centrifuged to remove the remaining large aggregates. ${ }^{35,36}$ Dynamic light scattering (Note S1, Figure S1 and Table S1) and scanning electron microscopy measurements (Figure S2) show that the resulting translucent solution consists of particles with an average size of $\sim 150 \mathrm{~nm}$. X-ray photoelectron spectroscopy (Figure S3) on the sonicated samples shows the presence of $\mathrm{N}_{1 \mathrm{~s}}$ peaks at $401.1 \mathrm{eV}$ and 398.8 $\mathrm{eV}$ and $\mathrm{K}_{2 \mathrm{p}}$ peak at $293 \mathrm{eV}$, verifying the presence of both the $\mathrm{NCN}^{-}$terminating group and $\mathrm{K}^{+}$ions in agreement with previous reports on the bulk sample. ${ }^{31}$ Such sonicated samples are often referred as exfoliated layer structures. ${ }^{35}$ However, the particles studied herein, whilst forming a stable suspension, are still too large to be considered as 2D structures of a few atomic layers. ${ }^{37}$ The sonicated samples used here were found to possess similar properties as the bulk, 
unsonicated (and more scattering) samples, evidenced by their indistinguishable transient absorption decay kinetics (Figure S4).

Figure $1 \mathrm{~b}$ shows the absorption spectra of the sonicated suspension $\left({ }^{\mathrm{NCN}} \mathrm{CN}_{\mathrm{x}}, 1 \mathrm{mg} / \mathrm{ml}\right.$ in water) which exhibits a characteristic absorption band edge at $\sim 450 \mathrm{~nm}(2.75 \mathrm{eV})$. This is consistent with previous DFT calculations, which predict a delocalized band structure of amino terminated $\mathrm{CN}_{\mathrm{x}}$ with a direct bandgap of $\sim 2.7 \mathrm{eV} .{ }^{18}$ The apparent absorption tail beyond $450 \mathrm{~nm}$ is attributed to Rayleigh scattering by the particles. Consistent with this, the PL of the ${ }^{\mathrm{NCN}} \mathrm{CN}_{\mathrm{x}}$ samples shows a main peak at $455 \mathrm{~nm}$, with a slight shoulder at $475 \mathrm{~nm} .{ }^{12}$

Similar to previous studies, ${ }^{32,33}$ simulated solar irradiation (AM 1.5G, 1 Sun) of a yellow ${ }^{{ }^{C N}}{ }^{C N_{x}}$ dispersion in the presence of 4-MBA (10 mM) for $10 \mathrm{~min}$ under Argon produced a blue coloration of the sample (Figure 1c, left). The blue color can persist for hours or even days in an anaerobic environment. The associated change of the absorption spectrum demonstrates both a bleach signal (full range data shown in Figure S5) and a characteristic photoinduced absorption (PIA), which is peaked at $680 \mathrm{~nm}$ and has broad and featureless absorption extending to the near IR (Figure 1d). This PIA feature is assigned to electron accumulation in ${ }^{\mathrm{NCN}} \mathrm{CN}_{\mathrm{x}}$, by both our results (vide infra) and by previously reported experiments where addition of proton reduction catalysts in the dark led to the loss of the blue color and the

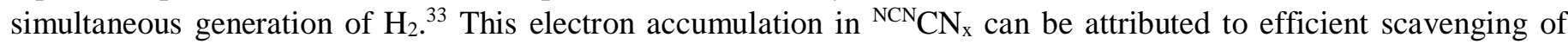
photogenerated holes by the 4-MBA (Scheme S1). In the presence of oxygen, no blue coloration can be detected, indicating that oxygen can act as an electron acceptor, as previously reported. ${ }^{38-40}$ For this reason, all measurements reported herein were conducted in an anaerobic environment unless otherwise noted. The bleach signal can be either attributed to the state-filling effects of band edge excitons as widely observed in quantum dots or a simple ground-state bleach as in a molecular picture. Investigation of the growth kinetics of the PIA feature at $680 \mathrm{~nm}$ indicates that the electron accumulation is a slow process (timescale of mins under one sun illumination) with its kinetics depending on both the 4-MBA concentration and the illumination light intensities (Figure S6), as we will discuss below.

\section{Photophysical studies of ${ }^{\mathrm{NCN}} \mathrm{CN}_{\mathrm{x}}$ in solution and their fluence dependent dynamics}

(a)

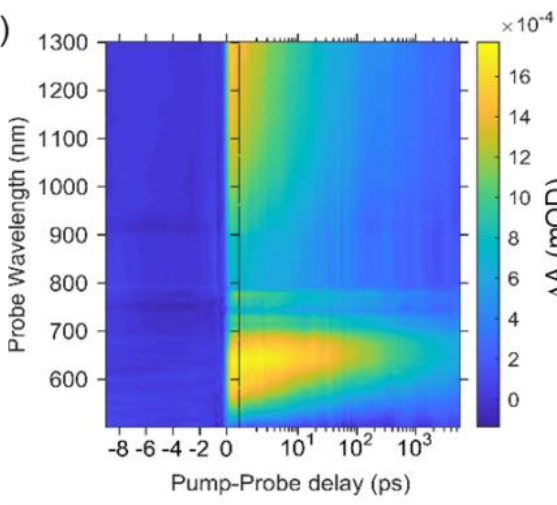

(b)

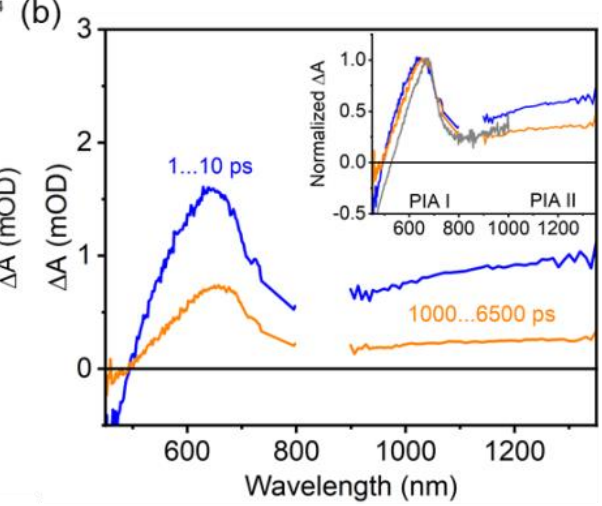

(c)

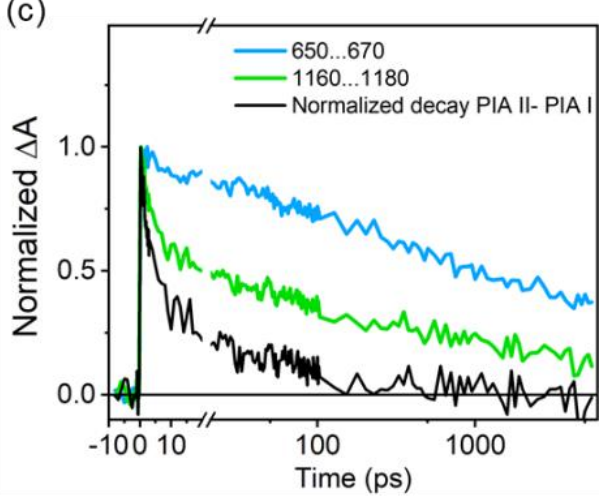

(d)

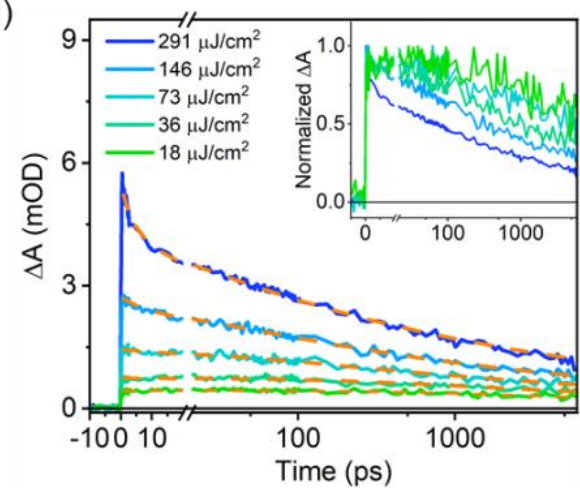

(e)

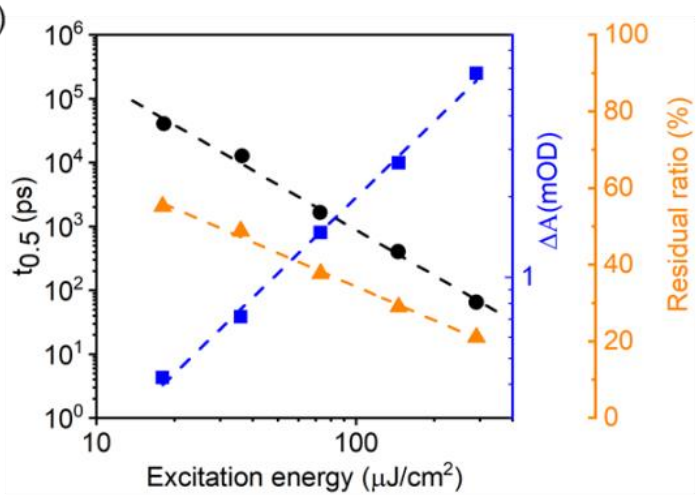

Figure 2. Transient absorption spectra of ${ }^{\mathrm{NCN}} \mathrm{CN}_{\mathrm{x}}$ dispersed in water $(1 \mathrm{mg} / \mathrm{ml})$ in the absence of 4-MBA and its fluence-dependent electron recombination kinetics. (a) Transient absorption spectra of ${ }^{\mathrm{NCN}} \mathrm{CN}_{\mathrm{x}}$ measured up to $6 \mathrm{~ns}$ time delay after excitation at $355 \mathrm{~nm}, \sim 73 \mu \mathrm{J} / \mathrm{cm}^{2}$. (b) Comparison of the transient spectra averaged from 1 to $10 \mathrm{ps}$ and from 1000 to 6500 ps. Intermediate spectra are shown in Figure S7 for clarity. Inset: the corresponding normalized difference spectra; the grey curve is taken from the inset of Figure 1c of the Argon sample. (c) Comparison of decay kinetics averaged from $660 \mathrm{~nm}$ to $680 \mathrm{~nm}$, assigned to photogeneration electrons, and from $1160 \mathrm{~nm}$ to $1180 \mathrm{~nm}$. The 
black curve is the difference in the kinetics by normalizing both at $6 \mathrm{~ns}$ and subtract the former from the latter. (d) Excitation fluence dependent electron decays kinetics monitored at $680 \mathrm{~nm}$. The overlaid orange dashed curves are fits from the power-law decays. Inset: the normalized data. (e) Dependence of the initial amplitude $(\boldsymbol{\bullet})$, decay half time $(\bullet)$ and $\%$ of residual signal $(\triangle)$ at $6 \mathrm{~ns}$ relative to initial signal for the photogenerated electron signal at $680 \mathrm{~nm}$ as a function of excitation energy determined from the data in Figure 2d.

Frist, we employed ultrafast transient absorption spectroscopy (TAS) to investigate the photophysical processes of ${ }^{\mathrm{NCN}}{ }^{\mathrm{CN}} \mathrm{N}_{\mathrm{x}}$ dispersed in water without hole scavenger (Figure 2a). Two transient PIA bands are resolved after excitation, namely a visible band peaking at $680 \mathrm{~nm}$ (PIA I) and a broader band in the near IR range from 900 to $1300 \mathrm{~nm}$ (PIA II) ${ }^{26}$ The spectral shape in the PIA I region agrees with the spectra of accumulated electrons obtained under steady-state irradiation with 4-MBA (Inset in Figure 2b, the grey line) and is therefore assigned to the PIA of electrons in ${ }^{{ }^{N C N}} \mathrm{CN}_{\mathrm{x}}$ following excitation. Similar electron absorption in the UV-Vis region has also been previously assigned for the melonbased $\mathrm{CN}_{\mathrm{x}}$ with amine terminations. ${ }^{12,26,41}$ Comparison of the decay kinetics in PIA I and PIA II region shows that the decay kinetics in the PIA II region are significantly faster than the PIA I region (Figure 2c). Detailed kinetic analysis indicates that the PIA II is contributed from the superposition of the above-mentioned electron signals and a faster decay component, with a half-life $\sim 4$ ps at $73 \mu \mathrm{J} / \mathrm{cm}^{2}$ (See Note S2 and Figure S8). The physical origin of this fast phase remains unclear. PIA signals in this spectral region have been previously attributed to be from the overlap of both electrons and hole signals in $\mathrm{CN}_{\mathrm{x}} \cdot{ }^{26}$ However, as shown in Figure S8 and discussed in Note S2, this interpretation is unsatisfying in the current system, where our data indicates a more complicated photophysical origin of PIA II band, which still requires further clarification. As the study herein focuses on the electron dynamics and their correlation with $\mathrm{H}_{2}$ generation, we therefore concentrate hereafter only on the electron decay measured in the PIA I region.

Figure $2 \mathrm{~d}$ shows the decay kinetics of the electron signal at $680 \mathrm{~nm}$ as a function of excitation fluence from $18 \mu \mathrm{J} / \mathrm{cm}^{2}$ to $291 \mu \mathrm{J} / \mathrm{cm}^{2}$. The initial $\Delta \mathrm{A}$ amplitude ( $300 \mathrm{fs}$ ) is found to increase linearly with excitation fluence, with a slope near unity $(\sim 0.94$, Figure $2 \mathrm{e})$, indicating that the efficiency of initial electron generation is independent of excitation fluence. However, the normalized decay kinetics show a strong fluence dependence (Inset in Figure 2d) with the half time, $t_{0.5}$, decreasing from $4500 \mathrm{ps}$ at $18 \mu \mathrm{J} / \mathrm{cm}^{2}$ to only $100 \mathrm{ps}$ at $291 \mu \mathrm{J} / \mathrm{cm}^{2}$ (Figure $2 \mathrm{e}$ ). In the absence of scavengers, the decay of electrons in excited photocatalysts can normally be attributed to electron trapping, electron-hole bimolecular recombination or trap-assisted electron-hole recombination. We found the decay kinetics can be well-fitted by a power-law relationship: $\mathrm{A} \propto t^{-\beta}$, similar to previous results, ${ }^{12,27,42}$ with $\beta$ lying within $0.20 \pm 0.02$ for all decays (Figure 2d, with more details shown in Note S3, Figure S9 and Table S2). Such fluence-dependent, power-law decay kinetics have also been widely observed in the study of bimolecular recombination of both metal oxides such as $\mathrm{TiO}_{2}{ }^{4}$ and organic semiconductor blends. ${ }^{12,27}$ An exponential distribution of tail states, resulting in trapping/detrapping mediated recombination has been suggested to account for the dispersive nature of this bimolecular recombination (i.e., $\beta<1) .{ }^{43-45}$ As further discussed below, these results indicate the existence of tail states and analogous trapping/detrapping mediated recombination processes in the ${ }^{{ }^{N C N}} \mathrm{CN}_{\mathrm{x}}{ }^{12,46} \mathrm{We}$ observe that the residual electron signal at $6 \mathrm{~ns}$ decreases from 55\% to $20 \%$ of the initial amplitude when the excitation power increased from $18 \mu \mathrm{J} / \mathrm{cm}^{2}$ to 290 $\mu \mathrm{J} / \mathrm{cm}^{2}$, indicating a larger loss of photogenerated electrons through faster bimolecular recombination at higher fluences. These results highlight that the recombination kinetics, and the resulting proportion of long-lived electrons, are highly dependent on the concentration of photogenerated charges in ${ }^{\mathrm{NCN}} \mathrm{CN}_{\mathrm{x}}$. 
Electron accumulation (or $\mathrm{N}$-type doping) achieved in the ${ }^{\mathrm{NCN}} \mathrm{CN}_{\mathrm{x}}$ in the presence of 4-MBA during the ultrafast measurement and its impact on charge dynamics
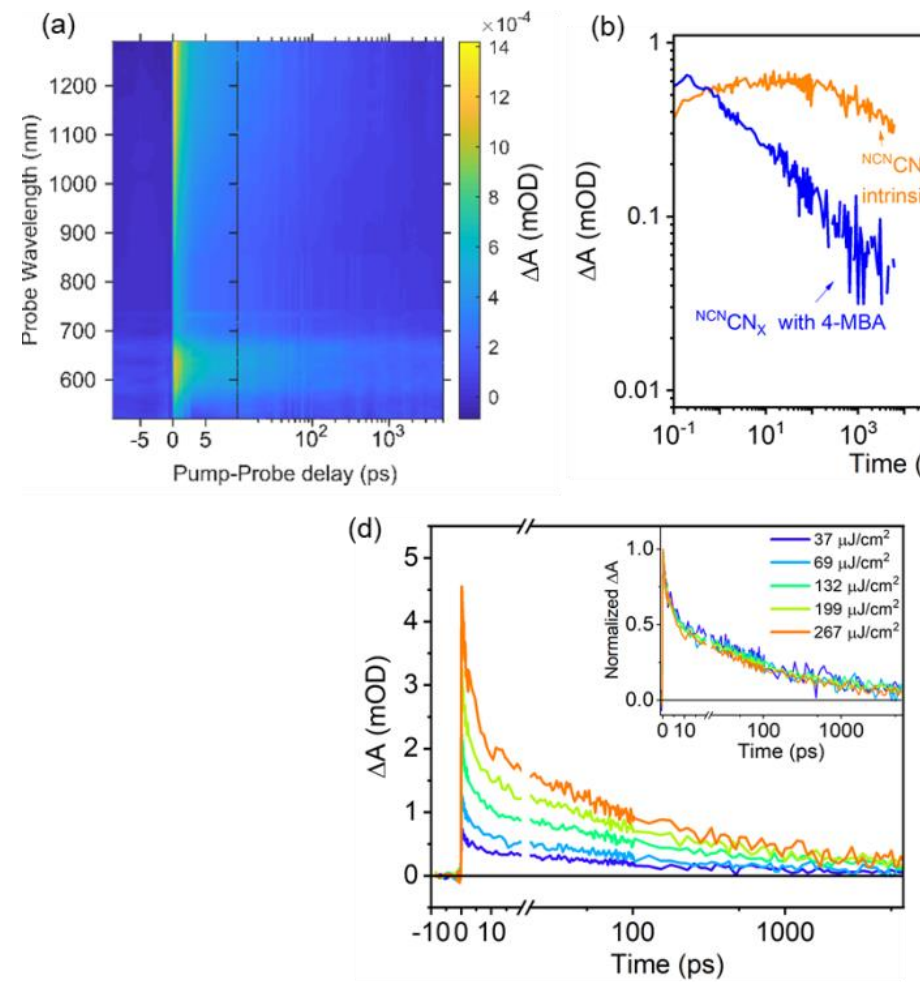
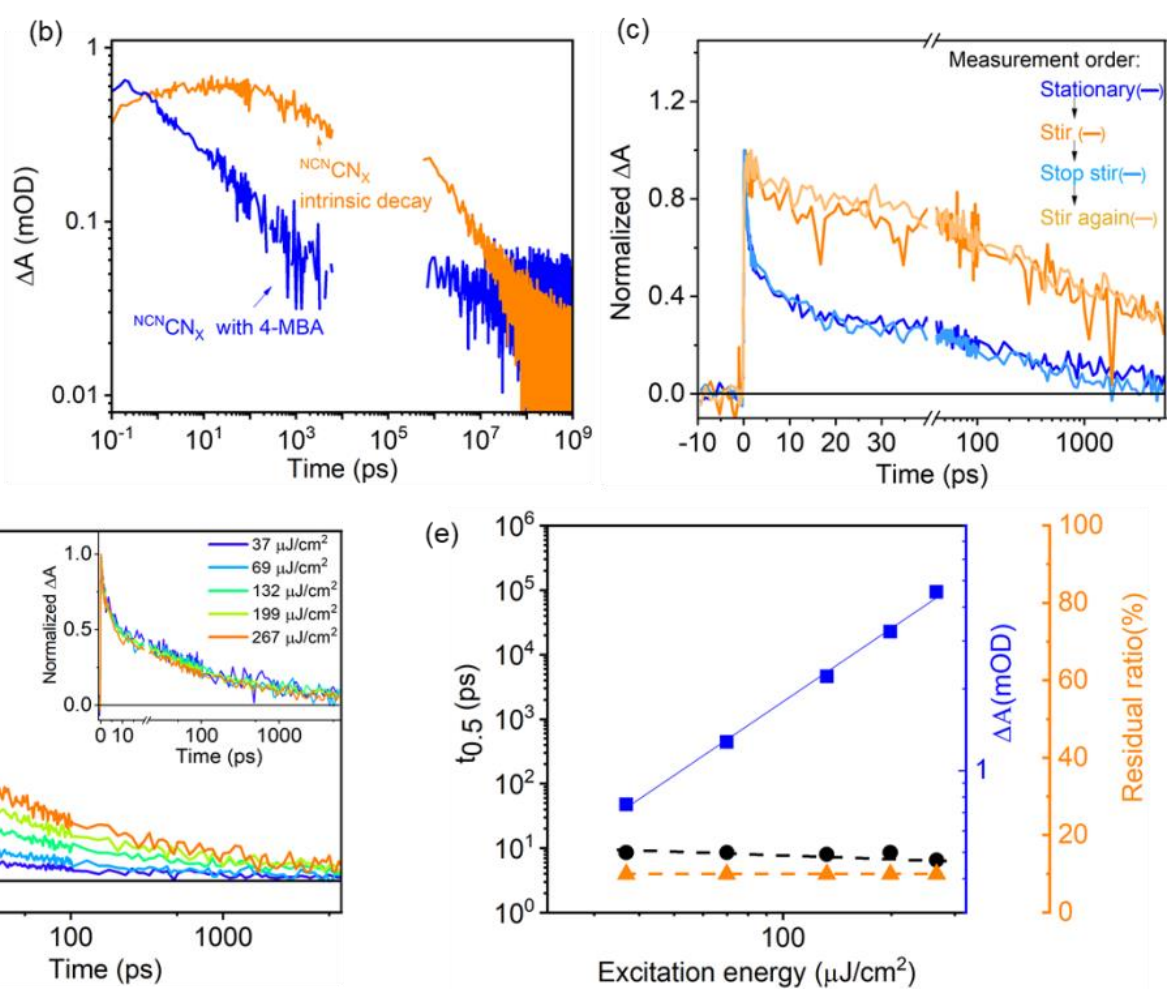

Figure 3. Electron accumulation in ${ }^{\mathrm{NCN}} \mathrm{CN}_{\mathrm{x}}$ induced by the addition of 4-MBA, as measured by its impact on the electron decay kinetics. (a) Transient absorption spectra of ${ }^{\mathrm{NCN}} \mathrm{CN}_{\mathrm{x}}$ in the presence of 4-MBA $(10 \mathrm{mM})$ at different time delays after excitation at $355 \mathrm{~nm}$. (b) Comparison of the corresponding electron decay kinetics measured at 680 $\mathrm{nm}$ with and without 4-MBA. The kinetic traces over 9 orders of magnitude from fs to ms are obtained by combining both ultrafast and microsecond TAS measurements excited at the same laser intensity. (c) Effect of stirring on the electron decay kinetics of ${ }^{\mathrm{NCN}} \mathrm{CN}_{\mathrm{x}}$ in the presence of $10 \mathrm{mM}$ 4-MBA. Measurements are conducted in the following orders: Stationary, with stirring, stop stirring and then stirring again. Stirring had no effect on equivalent data for the ${ }^{\mathrm{NCN}} \mathrm{CN}_{\mathrm{x}}$ sample without 4-MBA (Figure S13). Data measured at $\sim 69 \mu \mathrm{J} / \mathrm{cm}^{2}$ (d) Fluence dependent electron decay kinetics of ${ }^{\mathrm{NCN}} \mathrm{CN}_{\mathrm{x}}$ in the presence of 4-MBA, measured without stirring. Inset graph: the normalized kinetics. (e) Dependence of the initial amplitude $(\bullet)$, decay half time $(\bullet)$ and $\%$ of residual signal $(\triangle)$ relative to initial signal for the photogenerated electron signal at $680 \mathrm{~nm}$ as a function of excitation energy determined from the data in Figure 3(d). For (a) to (c), the pulse energy is controlled within $37 \mu \mathrm{J} / \mathrm{c} \mathrm{m}^{2}$ to $70 \mu \mathrm{J} / \mathrm{cm}^{2}$.

With the above spectral assignment of the ${ }^{\mathrm{NCN}} \mathrm{CN}_{\mathrm{x}}$ samples and its decay behavior, we now focus on the electron accumulation and dynamics in ${ }^{\mathrm{NCN}} \mathrm{CN}_{\mathrm{x}}$ in the presence of 4-MBA during the pulsed TAS measurement. Figure 3a shows the ultrafast transient absorption spectra of the ${ }^{{ }^{N C N}} \mathrm{CN}_{x}$ in the presence of $10 \mathrm{mM}$ 4-MBA. The spectra (Figure S10) show almost identical spectral shape to those in the absence of 4-MBA (Figure 2b), demonstrating that photogenerated electrons are again being monitored. However, the decay kinetics of these electrons is dramatically accelerated by the addition of 4-MBA (Figure 3b), with the decay half-times of $3000 \mathrm{ps}$ and $10 \mathrm{ps}$ for the system without and with addition of 4-MBA, respectively. The addition of other commonly used hole scavengers, triethanolamine and methanol, was found to lead to analogous accelerations of the electron decay kinetics (Figure S11). As we demonstrate below, such accelerated electron decay kinetics arise from faster charge recombination kinetics triggered by electron accumulation in the presence of hole scavenger. This was first evidenced by the appearance of a blue spot in the sample suspension at the point of laser excitation, directly indicating the existence of electron accumulation during the fs measurement (Figure S12, See Note S4 for further discussion). More importantly, stirring of the solution led to essentially identical decay kinetics in the presence and absence of 4-MBA (Figure 3c). Stirring cannot be expected to impede hole scavenging by 4-MBA but does disperse ${ }^{\mathrm{NCN}} \mathrm{CN}_{\mathrm{x}}$ particles containing accumulated electrons throughout the sample solution. Further control experiments show that stirring does not affect the decay kinetics of the ${ }^{\mathrm{NCN}} \mathrm{CN}_{\mathrm{x}}$ in the absence of 4-MBA (Figure S13). The possibility of photodegradation of ${ }^{{ }^{N C N}} \mathrm{CN}_{\mathrm{x}}$ at the laser excitation port is therefore excluded. Additional control experiments show that pre-illumination of the ${ }^{{ }^{N C N}} \mathrm{CN}_{\mathrm{x}}$ samples with 4-MBA under continuous simulated solar 
illumination (i.e., turning the suspension completely blue as in Figure 1c) produces identical decay kinetics at $680 \mathrm{~nm}$ to those measured for ${ }^{\mathrm{NCN}} \mathrm{CN}_{\mathrm{x}}$ in the presence of 4-MBA without stirring (Figure S14). These above results therefore confirm unambiguously that the accelerated electron decay observed in the presence of 4-MBA results from the photoinduced electron accumulation, rather than resulting directly from an ultrafast scavenging process. The PL decays of excitons also provide evidence of faster charge recombination when electrons are accumulated in ${ }^{\mathrm{NCN}} \mathrm{CNx}$ (Figure S15). To the authors' knowledge, this is the first reported observation of charge accumulation in ultrafast spectroscopy studies of carbon nitride photocatalysts and the resultant dramatic acceleration of electron recombination kinetics.

As demonstrated below, this finding of accelerated charge recombination kinetics under conditions of charge accumulation are important not only in terms of the correct interpretation of the pulsed spectroscopic studies, but also as they provide new insights regarding the light intensity dependence of photocatalytic performance under continuous irradiation. This electron accumulation is consistent with the fact that the $500 \mathrm{~Hz}$ repetition frequency of the pump laser is much faster than the previously characterized $\sim \mathrm{s}$ time scale electron extraction from the ${ }^{\mathrm{NCN}} \mathrm{CN}_{\mathrm{x}}{ }^{32} \mathrm{On}$ the other hand, although not resolved directly, the hole extraction is estimated in the order of ns to $\mu$ s timescale (Figure S16), consistent with a previous study. ${ }^{12}$ Therefore, in the presence of 4-MBA, electrons can be accumulated in ${ }^{{ }^{N C N}{ }^{2} N_{x}}$ during the ultrafast laser measurements due to unbalanced hole and electron extraction kinetics, resulting in the remarkable $\sim 400$ fold acceleration of electron decay kinetics.

We now turn to consider the excitation fluence dependence of the electron decay kinetics at $680 \mathrm{~nm}$ in the presence of 4-MBA and under these charge accumulation conditions (Figure 3d and 3e). Similar to the data in the absence of 4MBA (Figure 2e), the initial amplitude of the electron signals still increases linearly with excitation fluence (Figure 3e) even under charge accumulation. However, the electron decay kinetics in the presence of 4-MBA show strikingly almost no fluence dependence, in all cases exhibiting a decay half-time of $\sim 10$ ps (Figure $3 \mathrm{~d}$ Inset). This is substantially faster than the fastest decay kinetics observed in the absence of 4-MBA (100 ps). Consequently, only around 5\% of photogenerated electrons survive to $6 \mathrm{~ns}$ for samples with 4-MBA regardless the excitation fluence. Combining these ultrafast data with analogous microsecond TAS measurements (Figure 3b), it is apparent that these residual electrons do not decay significantly up to ms timescales and should therefore be assigned to the long-lived electrons potentially capable of driving $\mathrm{H}_{2}$ production.

The fast, and fluence independent, electron decay dynamics observed under electron accumulated in ${ }^{\mathrm{NCN}} \mathrm{CNx}$ suggest that the steady-state density of electrons accumulated in these experiments is much greater than those generated by a single pulse in the absence of 4-MBA, even for the highest excitation densities used herein. We quantify the absorption of the accumulated electrons in the beam path of the ultrafast TAS system by using it as an in situ double beam UV/vis spectrometer (see Note S5 in SI). The absorption changes at $680 \mathrm{~nm}$ was measured to be $70 \mathrm{mOD}$, corresponding to an accumulated electron density of $4 \times 10^{17} \mathrm{~cm}^{-3}$, assuming an extinction coefficient of electrons to be $1000 \mathrm{M}^{-1} \mathrm{~cm}^{-1} 47$ (Note S6). In contrast, the measured maximal TAS signal at an excitation density of $\sim 267 \mu \mathrm{J} \mathrm{cm}^{-2}$ is only $4.5 \mathrm{mOD}$, corresponding to an electron density of $2.5 \times 10^{16} \mathrm{~cm}^{-3}$. These results confirm that under conditions of electron accumulation in the presence of 4-MBA, accumulated electrons are in large excess compared to photogenerated electron/holes densities by a single laser pulse. Therefore, using semiconductor nomenclature, the ${ }^{\mathrm{NCN}} \mathrm{CN}_{\mathrm{x}}$ is heavily $\mathrm{n}$ doped by irradiation when adding 4-MBA into the suspension. 
Relationship between the electron accumulation (N-doping) amount in ${ }^{\mathrm{NCN}} \mathrm{CN}_{\mathrm{x}}$ and its carrier decay dynamics
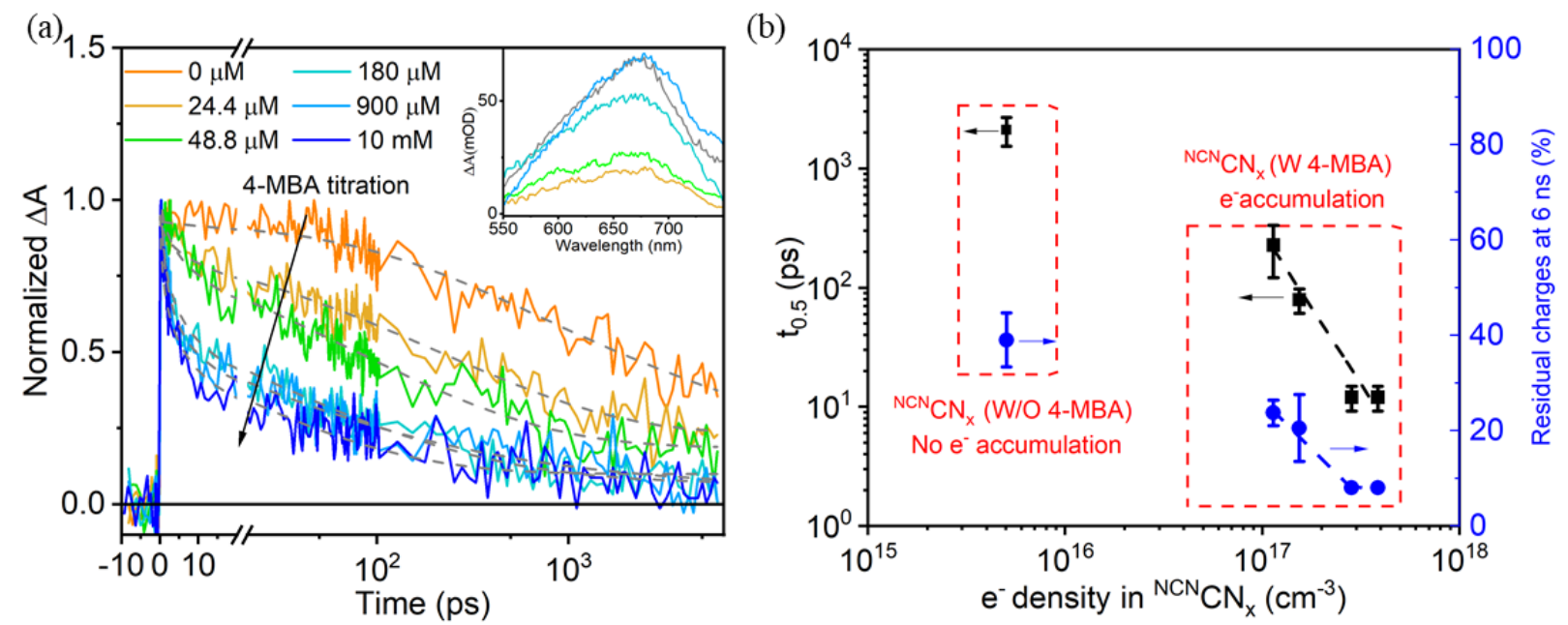

Figure 4. Electron recombination kinetics as a function of 4-MBA concentration. (a) Electron recombination kinetics probed at $680 \mathrm{~nm}$ when varying the [4-MBA] in the ${ }^{{ }^{N C N}}{ }^{2} N_{x}$ solution. The dashed lines represent the stretched exponential fits by Eq 1. Inset figure: the differential absorption measured in situ at the laser excitation spot before and after the fs laser measurement (details in SI Note 4). The solid grey curve in the Inset figure is the electron spectrum taken from Figure 1c (Light, argon) and scaled. (b) Correlation between electron accumulation density in ${ }^{\mathrm{NCN}} \mathrm{CN}_{\mathrm{x}}$ and their decay half-time under different 4-MBA concentrations. The accumulated electron densities are calculated by taking the steady-state difference absorption shown in the inset of Figure $4 \mathrm{a}$ and applying the Lambert-Beer relationship with the assumption of electron extinction to be $1000 \mathrm{M}^{-1} \mathrm{~cm}^{-1} .{ }^{47}$ Excitation density for all the samples: $\sim 34-37 \mu \mathrm{J} / \mathrm{cm}^{2}$.

To further quantify the relationship between electron accumulation and charge recombination kinetics, we undertook ultrafast TAS measurement as a function of 4-MBA concentration. A variation of the 4-MBA concentration is expected to change the rate of hole scavenging and therefore affect the concentration of accumulated electrons in ${ }^{\mathrm{NCN}} \mathrm{CN}_{\mathrm{x}}$. Indeed, as shown in the inset Figure 4a, an increase of 4-MBA concentration leads to an increase in the amplitude of the steadystate $\Delta A$ at $680 \mathrm{~nm}$ generated in situ by the laser excitation beam. This blue state absorption saturates when the 4-MBA concentration is around $0.9 \mathrm{mM}$, similar to the saturation concentration observed under continuous Xenon lamp irradiation (Figure S18). This indicates the concentration where the hole extraction rate is no longer the yield determining step in the electron accumulation. Kinetically, increasing the concentration of 4-MBA from $24.4 \mu \mathrm{M}$ to 900 $\mu \mathrm{M}$ is found to lead to a decrease of the electron decay half time from $\sim 300 \mathrm{ps}$ to $\sim 10 \mathrm{ps,}$ all significantly faster than the $\sim 3000$ ps observed in the absence of any 4-MBA at this fluence. By calculating the accumulated electron density through the in-situ absorption changes and applying the Beer-Lambert law, we correlate the decay half time with the accumulated electron densities in the Figure 4b. At all 4-MBA concentrations tested, the accumulated electron density far exceeds the one pulse photogenerated electron density, $5 \times 10^{15} \mathrm{~cm}^{-3}\left(\sim 34 \mu \mathrm{J} / \mathrm{cm}^{2}\right.$ excitation). As such, pseudo-first order conditions (i.e. electrons in far excess compared to holes) should apply, resulting, in the presence of trapping/detrapping mediated recombination, in stretched exponential kinetics. ${ }^{43}$ Consistent with this expectation, the electron decay kinetics in the presence of 4-MBA were observed to follow stretched exponential decay behavior (Eq 1 ), with the decay half time increasing with the carrier density following the equation (Eq 2) ${ }^{48}$ :

$$
\begin{array}{ll}
\mathrm{A}=\mathrm{A}_{0} \exp \left(\left(-\frac{t}{t_{0}}\right)^{\beta}\right) & \text { Eq } 1 . \\
t_{0.5} \propto[n]^{-\alpha} \text { and } \alpha=\frac{1}{\beta} & \text { Eq } 2 .
\end{array}
$$

where $n$ is the accumulated electron density. As shown in Figure 4a, stretched exponential fits are found to show excellent agreement with the experimental results, resulting in a $\beta$ value $\sim 0.15$ to 0.4 (Table S3) depending on the 4MBA concentration. Log/Log plots of $t_{50 \%}$ versus [ $n$ ] yield a straight-line behavior, consistent with equation 2 , with $\alpha$ $\sim 2.5$ (Figure $4 \mathrm{~b}$ ) in reasonable agreement with the fits to Figure $4 \mathrm{a}$. We therefore conclude that the electron decay kinetics measured both in the presence and absence of 4-MBA are consistent with dispersive recombination kinetics. This dispersive behavior most likely originates from charge trapping/detrapping, and the change from power law to 
stretched exponential behavior results from the change of conditions where $\left[e^{-}\right] \approx\left[h^{+}\right]$(power-law) to $\left[e^{-}\right] \gg\left[h^{+}\right]$ (pseudo-first order; stretched exponential) in the presence of 4-MBA.

From a functional viewpoint, the most striking aspect of the above results is the rapid acceleration of charge recombination kinetics caused by photoinduced electron accumulation in the presence of a hole scavenger. We consider below the impact of this upon the efficiency of $\mathrm{H}_{2}$ evolution under steady-state operation.

\section{Implication of the charge accumulation on the photocatalytic $\mathrm{H}_{2}$ production performance}

(a)

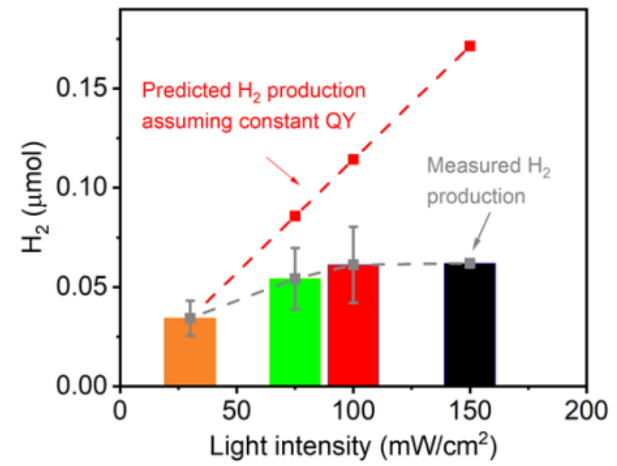

(b)

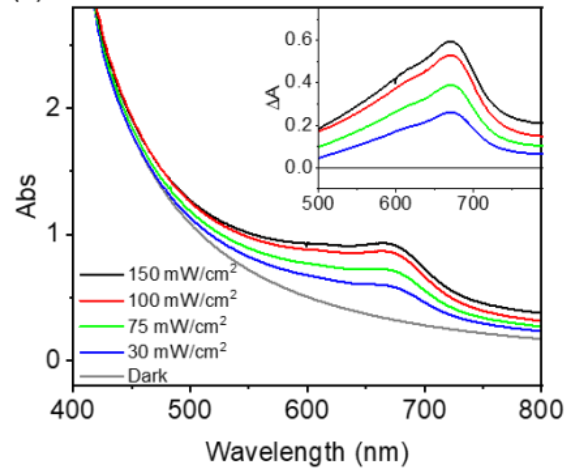

(c)

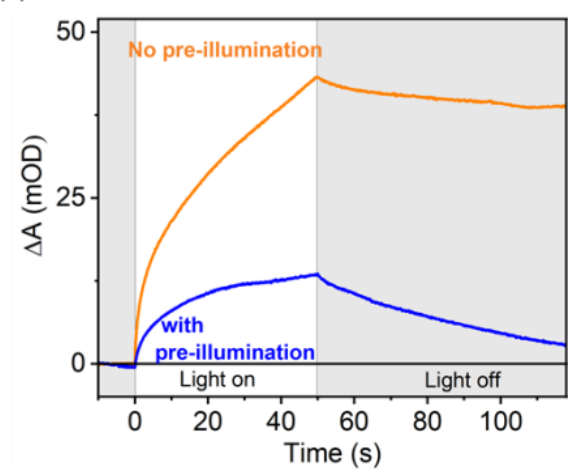

Figure 5. Irradiation fluence dependent $\mathbf{H}_{2}$ production of ${ }^{\mathrm{NCN}} \mathrm{CN}_{\mathbf{x}}$. (a) $\mathrm{H}_{2}$ production measured in ${ }^{\mathrm{NCN}} \mathrm{CN}_{\mathrm{x}}$ using simulated solar irradiation (AM $1.5 \mathrm{G}, 25^{\circ} \mathrm{C}$ ) with and without neural density filters to generate varied light intensities (30 to $150 \mathrm{~mW} / \mathrm{cm}^{2}$ ). Measurement time at each intensity: $1 \mathrm{~h}$. Red dashed line: predicted amount of $\mathrm{H}_{2}$ assuming a constant quantum yield for $\mathrm{H}_{2}$ production (same value as $30 \mathrm{~mW} / \mathrm{cm}^{2}$ ). (b) UV-vis absorption of samples taken after 3 min light illumination. (c) the photoinduced steady-state absorption (PIA) changes of the samples at $660 \mathrm{~nm}$ with and without pre-irradiation. The pre-irradiation samples are 4-MBA containing samples which were first illuminated for few min under the continuous illumination (100 W Quartz Tungsten Halogen lamp). After turning blue, indicating electron accumulation in the system, the samples are then subjected to the PIA measurements. Conditions (a-c): 4-MBA (10 $\mathrm{mM}), \mathbf{N i P}(15 \mu \mathrm{M})$ and ${ }^{\mathrm{NCN}} \mathrm{CN}_{\mathrm{x}}(1 \mathrm{mg} / \mathrm{ml})$ in aqueous potassium phosphate solution (KPi; $\left.0.1 \mathrm{M}, \mathrm{pH} 4.5,2 \mathrm{~mL}\right)$.

Figure 5a shows the amounts of $\mathrm{H}_{2}$ production from ${ }^{\mathrm{NCN}} \mathrm{CN}_{\mathrm{x}}$ aqueous solutions under different illumination intensities with 4-MBA as the hole scavenger and a molecular DuBois-type $\mathrm{H}_{2}$ production catalyst NiP. ${ }^{49}$ Due to the slow electron extraction kinetics from ${ }^{\mathrm{NCN}} \mathrm{CN}_{\mathrm{x}}$ by $\mathbf{N i P}$, as we have reported previously, ${ }^{32,50}$ the presence of NiP is expected to have negligible impact on charge accumulation and associated accelerated charge recombination kinetics discussed above on the ps-ns time-scale (also confirmed in Figure S19). We found that increasing the light intensity from $30 \mathrm{~mW} / \mathrm{cm}^{2}$ to $150 \mathrm{~mW} / \mathrm{cm}^{2}$ only doubles the amount of $\mathrm{H}_{2}$ production from $0.032 \mu \mathrm{mol} / \mathrm{h}$ to $0.061 \mu \mathrm{mol} / \mathrm{h}$. This indicates that the apparent quantum yield is about 3-fold larger at $30 \mathrm{~mW} / \mathrm{cm}^{2}$ as compared to $150 \mathrm{~mW} / \mathrm{cm}^{2}$. The photocatalytic solutions turned blue during the measurements, with $\Delta \mathrm{A}$ at $680 \mathrm{~nm}$ measured to be 0.2 and 0.6 for $30 \mathrm{~mW} / \mathrm{cm}^{2}$ and $150 \mathrm{~mW} / \mathrm{cm}^{2}$ after 3 min illumination (Figure 5b), respectively. In other words, 3-fold more electrons accumulate under the higher illumination intensity. As shown in Figure 2d, the increase of light intensity can cause faster bimolecular recombination between the photogenerated charge pairs. However, this acceleration is considerably smaller than the dramatic increase of charge recombination due to electron accumulation. Therefore, the lower quantum yield at higher light intensities is more likely due to the electron-accumulation induced recombination loss.

To confirm the impact of electron accumulation on the quantum yield of $\mathrm{H}_{2}$ generation, we performed photo-induced absorption spectroscopy (PIAS) measurements on the system, using a square-wave modulated LED (365 $\mathrm{nm}$ ) to excite the system whilst continuously monitoring the sample absorption at $660 \mathrm{~nm}$. Without the addition of 4-MBA, no optical changes were observed during the light on and off periods (Figure S20), agreeing with fast electron-hole recombination of this system, $\mathrm{t}_{1 / 2} \sim$ ps to $\mathrm{ns}$, impeding $\mathrm{H}_{2}$ production. With 4-MBA and NiP, light illumination is found to lead to a continuous absorption increase at $660 \mathrm{~nm}$ on the timescale of tens of seconds (Figure 5c), indicating the slow accumulation of electrons in the system. When light is turned off, these accumulated charges decay slowly, most likely transferring to NiP to produce $\mathrm{H}_{2}$ as reported previously. ${ }^{12}$ In contrast, when pre-illuminating the samples to form the electron-accumulated blue state, the photoinduced absorption changes under the same excitation power is reduced to only a third. These results therefore quantitatively demonstrate that electron accumulation can indeed impede further accumulation of photogenerated charges under operating conditions, which therefore limits the performance of the system. 
(a)

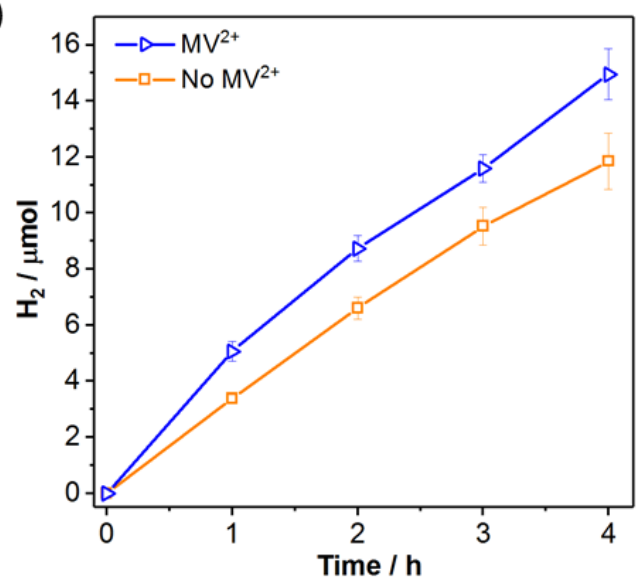

(b)

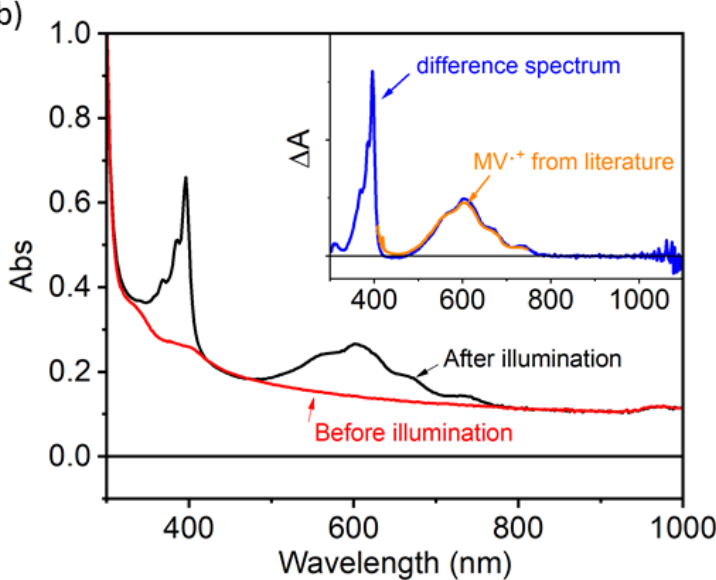

Figure 6. Photocatalytic $\mathrm{H}_{2}$ production with ${ }^{\mathrm{NCN}} \mathrm{CN}_{\mathrm{X}}$ and $\mathrm{NiP}$ in the presence and absence of $\mathrm{MV}^{2+}$ as an electron mediator. (a) Comparison of $\mathrm{H}_{2}$ production rate with and without $\mathrm{MV}^{2+}$. Conditions: ${ }^{{ }^{C N}} \mathrm{CN}_{\mathrm{x}}(5 \mathrm{mg}), \mathbf{N i P}(50 \mathrm{nmol})$, 4-MBA $(30 \mu \mathrm{mol})$ in $\mathrm{KPi}(0.1 \mathrm{M}, \mathrm{pH} 4.5,3 \mathrm{~mL})$, with and without $\mathrm{MV}^{2+}(50 \mu \mathrm{mol}), \mathrm{N}_{2}$, AM 1.5G, 1 Sun. (b) Absorption spectra of the ${ }^{\mathrm{NCN}} \mathrm{CN}_{\mathrm{x}}$ solution with 4-MBA and $\mathrm{MV}^{2+}$ in the solution after illumination.

The electron accumulation observed in ${ }^{\mathrm{NCN}} \mathrm{CN}_{\mathrm{x}}$ under operating conditions in Figure $5 \mathrm{~b}$ arises from the slow electron transfer from ${ }^{\mathrm{NCN}} \mathrm{CN}_{\mathrm{x}}$ to NiP. One way to reduce this electron accumulation, and resultant increased charge recombination losses, is to extract electrons more quickly from the ${ }^{{ }^{N C N}}{ }^{C N} N_{x}$ by adding a redox relay that can shuttle electrons from the photocatalysts to the proton reduction catalyst. ${ }^{51,52}$ Here we used $\mathrm{MV}^{2+}\left(\mathrm{E}^{\circ \prime}=-0.45 \mathrm{~V}\right.$ vs NHE$)$ as an example redox relay, which has been previous applied in quantum dots to act a redox shuttle. ${ }^{53-55}$ The results show that the addition of $\mathrm{MV}^{2+}$ indeed leads to $~ 30 \%$ improvement in $\mathrm{H}_{2}$ production (Figure 6a). Figure $6 \mathrm{~b}$ shows the absorption spectral changes of ${ }^{\mathrm{NCN}} \mathrm{CN}_{\mathrm{x}}\left(1 \mathrm{mg} / \mathrm{ml}\right.$ with $10 \mathrm{mM}$ 4-MBA, in the absence of NiP) after adding $\mathrm{MV}^{2+}$ and after illumination. A characteristic absorption is observed in the range of 400 to $700 \mathrm{~nm}\left(\Delta A_{\max }=600 \mathrm{~nm}\right)$, in agreement with the previously reported spectra $\mathrm{MV}^{\bullet+}$ (overlaid orange trace ${ }^{52}$ ) and indicating photoinduced electron transfer from ${ }^{\mathrm{NCN}} \mathrm{CN}_{\mathrm{x}}$ (CB edge: $-1.1 \mathrm{~V}$ vs NHE) to $\mathrm{MV}^{2+}$. Furthermore, we also found that this reduced $\mathrm{MV}^{+}$can be quenched by the addition of NiP, indicating the active electron transfer from $\mathrm{MV}^{\bullet+}$ to NiP (Figure S21). However, we observed that the addition of $\mathrm{MV}^{2+}$ also led to the aggregation of ${ }^{{ }^{N C N}} \mathrm{CN}_{\mathrm{x}}$, presumably through ionic interactions ${ }^{56}$, which limited the further characterization of detailed charge transfer kinetics. Nevertheless, the above improvement of $\mathrm{H}_{2}$ production caused by addition of $\mathrm{MV}^{2+}$ clearly indicates that reducing charge accumulation in ${ }^{\mathrm{NCN}}{ }^{\mathrm{N}} \mathrm{N}_{\mathrm{x}}$ should be further explored to improve the performance of the ${ }^{\mathrm{NCN}} \mathrm{CN}_{x}$ photocatalyst. A recent study ${ }^{50}$ also showed that by using $2 \mathrm{D}$-(reduced) graphene oxide sheets to improve communication between ${ }^{\mathrm{NCN}} \mathrm{CN}_{\mathrm{x}}$ and $\mathbf{N i P}$, the $\mathrm{H}_{2}$ production of the system can also be improved. All these results indicate that further ways to facilitate electron extraction from ${ }^{{ }^{N C N}} \mathrm{CN}_{\mathrm{x}}$ to reduce the charge accumulation induced recombination would be vital to improve the performance of the ${ }^{\mathrm{NCN}} \mathrm{CN}_{\mathrm{x}}$ based photocatalysts.

The effects of electron accumulation on carrier recombination kinetics we report herein may be particularly dramatic due to the unusually long lifetime of accumulated electrons in ${ }^{\mathrm{NCN}} \mathrm{CN}_{\mathrm{x}}$. However, the general concept exemplified by this study, that imbalanced electron and hole extraction rates can result in accumulation of one type of charge and thereby accelerated recombination kinetics, is likely to be applicable to many photocatalytic systems. $\mathrm{CN}_{\mathrm{x}}$ synthesized from other precursors, such as urea, have also been reported to possess a large density of trapping states possibly related to pendant amino and urea groups, resulting in long-lived photogenerated trapped electrons (ms). ${ }^{12}$ We note that for urea synthesized $\mathrm{CN}_{\mathrm{x}}$, charge trapping appears to be energetically deeper, such that trapped electrons are unable to drive proton reduction, unlike the ${ }^{\mathrm{NCN}} \mathrm{CN}_{\mathrm{x}}$ studied herein. The charge accumulation is likely to be particularly important for water oxidation photocatalysts, where four electrons and four protons are involved in water oxidation reaction, resulting in a kinetically sluggish reaction, typically on the order of $\sim \mathrm{s}{ }^{1}$ This timescale is significantly slower than that of electron extraction using electron scavengers, typically from $\mathrm{ps}$ to $\mathrm{ms}$. As a result, photoinduced accumulation of holes can be expected for water oxidizing photocatalysts and may also result in accelerated recombination kinetics. Analogous hole accumulation has also been observed in water oxidation photoanodes, and shown to result in accelerated charge recombination losses (referred to as 'back electron/hole recombination'). ${ }^{11,57}$ Such accumulation driven recombination kinetics have been also reported in other systems. Gamelin et.al. ${ }^{58}$ has previously applied anaerobic photoexcitation of $\mathrm{CdSe} \mathrm{NCs}$ in the presence of a borohydride hole quencher, $\mathrm{Li}\left[\mathrm{Et}_{3} \mathrm{BH}\right]$, to yield colloidal n-type CdSe, with a characteristic electron absorption in the IR region, resulting in a clear (although relatively modest) acceleration of 
exciton decay times. Similarly, imbalanced electron and hole mobilities in organic solar cells have been shown to result in photoinduced charge accumulation and increased recombination losses. ${ }^{28}$

The photoinduced electron accumulation reported herein for ${ }^{{ }^{N C N}} \mathrm{CN}_{\mathrm{x}}$, and the resultant acceleration of charge recombination kinetics, is dependent upon the intensity of the incident irradiation, and is most severe for high light irradiances. This results in the efficiency of $\mathrm{H}_{2}$ generation decreasing with increasing irradiation intensity. Such intensity dependent efficiency will be important for operation under outdoor solar irradiation, and therefore for practical technological application, but is not widely considered in studies of photocatalysis. It is also important for consideration of the relevance of transient laser measurements, which often use relatively intense laser pulses. This accumulation is also dependent on the kinetics of hole scavenging, further emphasizing that the impact of this scavenging upon the desired functionality of proton reduction, and the danger of extrapolating from systems employing electron or hole scavengers to systems targeting the technologically desirable function of coupled water oxidation and reduction.

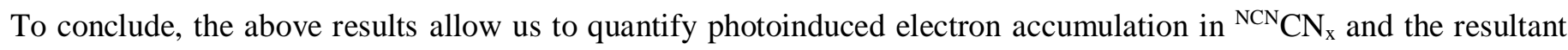
dramatic acceleration of the recombination kinetics in this photocatalyst. This charge accumulation results both from imbalanced electron and hole extraction as well as the long lifetime of trapped electrons in this system. This produces an acceleration of charge recombination in ${ }^{\mathrm{NCN}} \mathrm{CN}_{\mathrm{x}}$ by as much as 400 -fold. These results are found to be in at least qualitative agreement with the illumination intensity dependence of the $\mathrm{H}_{2}$ yield under simulated sunlight. Charge extraction by $\mathrm{MV}^{2+}$ as a redox mediator is demonstrated to show a promising performance improvement, attributed to the resultant faster electron extraction kinetics reducing electron accumulation. At the same time, it is important to note that, given their slow transfer kinetics to NiP, electron accumulation in ${ }^{\mathrm{NCN}} \mathrm{CN}_{\mathrm{x}}$ is necessary to drive a significant flux of $\mathrm{H}_{2}$ generation, such that electron accumulation in this system is both necessary for $\mathrm{H}_{2}$ generation but also results in enhanced recombination losses. As such, these results emphasize the importance of charge carrier kinetics, and their non-linear dependence upon irradiation intensity, in determining the efficiency of photocatalytic systems, and the importance of achieving reasonably balanced electron and hole extraction kinetics to achieve optimum photocatalytic efficiency.

\section{ASSOCIATED CONTENT}

\section{Supporting Information}

The Supporting Information is available free of charge on the ACS Publications website.

\section{Note S1 to Note S7; Table S1 to Table S3; Figure S1 to Figure 21;}

\section{Acknowledgement:}

Michael Sachs is acknowledged for his help with the ultrafast measurements. Dr. Xiaoe Li is acknowledged for her help with preparing the sonicated ${ }^{\mathrm{NCN}} \mathrm{CN}_{\mathrm{x}}$ samples. Prof. Jenny Nelson is acknowledged for her helpful discussions. This work was financially supported by the ERC AdG Intersolar grant (Grant No. 291482), the Christian Doppler Research Association (Austrian Federal Ministry for Digital and Economic Affairs and the National Foundation for Research, Technology and Development) and the OMV Group.

\section{Conflict of interests}

The authors declare no competing financial interest.

\section{Experimental sections}

\section{Synthesis of the ${ }^{\mathrm{NCN}} \mathrm{CN}_{\mathrm{x}}$}

Melamine was thermally polymerized at $550{ }^{\circ} \mathrm{C}$ for $4 \mathrm{~h}$ under air, forming benchmark amine terminated carbon nitride, ${ }^{\mathrm{H} 2 \mathrm{~N}} \mathrm{CN}_{\mathrm{x}}$. Bulk ${ }^{\mathrm{NCN}} \mathrm{CN}_{\mathrm{x}}$ was prepared by post-treatment of ${ }^{\mathrm{H} 2 \mathrm{~N}} \mathrm{CN}_{\mathrm{x}}$ with $\mathrm{KSCN}$ under argon atmosphere at $400{ }^{\circ} \mathrm{C}$ for $1 \mathrm{~h}$, then $500{ }^{\circ} \mathrm{C}$ for $30 \mathrm{~min}$, following published procedure. ${ }^{31,32}$

\section{Exfoliation process}

To produce a stable and well-dispersed solution for optical measurements, the bulk ${ }^{\mathrm{NCN}} \mathrm{CN}_{\mathrm{x}}$ suspension $(3 \mathrm{mg} / \mathrm{ml})$ was firstly sonicated in water overnight $(\sim 15 \mathrm{hr})$ to disperse the particles and then centrifuged at $3000 \mathrm{rpm}$ for $10 \mathrm{mins}$ to 
remove remaining large aggregates following a previously reported procedure. ${ }^{35}$ The solid content of the solution varies between 1.1 to $1.4 \mathrm{mg} / \mathrm{ml}$.

\section{Ultrafast measurement}

The fs-TAS were measured with a commercial transient absorption spectrometer (HELIOS, Ultrafast systems). The laser light was generated from the $1 \mathrm{kHz}$ Solstice (Newport Corp.) Ti: sapphire regenerative amplifier $(800 \mathrm{~nm})$ and was splitted to generate both the pump and the probe pulses. The pump light is passed through a TOPAS-Prime (Light Conversion Ltd.) optical parametric amplifier to generate the $355 \mathrm{~nm}$ excitation light. The energy of the excitation light was then adjusted by use of a natural density filter wheel. The probe light is produced by focusing the $800 \mathrm{~nm}$ light through a sapphire crystal ( $\sim 2 \mathrm{~mm}$ thickness). The time resolution of this setup was $200 \mathrm{fs}$. The samples are the as prepared exfoliated ${ }^{\mathrm{NCN}} \mathrm{CNx}$ samples $(\sim 1 \mathrm{mg} / \mathrm{ml}$ dispersed in water). The solution is kept stationary unless otherwise mentioned. All the solutions were purged with Argon in advance of the measurements.

\section{us-TAS measurement}

Microsecond to second transient absorption decays were acquired in diffuse reflectance mode with a previously reported setup. ${ }^{12}$ A Nd:YAG laser (OPOTEK Opolette $355 \mathrm{II}, 7 \mathrm{~ns}$ pulse width) was used as the excitation source and produced $355 \mathrm{~nm}$ light that was transmitted to the sample through a light guide. Typical excitation power densities of 180-360 $\mu \mathrm{J} / \mathrm{cm} 2$ and laser repetition rates of $1.1 \mathrm{~Hz}$ were used. The changes of reflectance observed were low $(<1 \%)$ and allowed for the transient signal to be taken as directly proportional to the concentration of excited state species. Probe light was generated by a $100 \mathrm{~W}$ Bentham IL1 quartz halogen lamp. Long pass filters (Comar Instruments) and an IR filter (H2O, $5 \mathrm{~cm}$ path length) were positioned between the lamp and sample to minimize short wavelength irradiation and heating of the sample. Diffuse reflectance from the sample was collected and relayed to a monochromator (Oriel Cornerstone 130) through a long pass filter to select the probe wavelength. Acquisitions were triggered by a photodiode (Thorlabs DET10A) exposed to laser scatter. Time-resolved intensity data was collected with a Si photodiode (Hamamatsu S3071). Data at times faster than $3.6 \mathrm{~ms}$ was amplified by custom electronics (Costronics) and recorded by an oscilloscope (Tektronics DPO3012) while data slower than $3.6 \mathrm{~ms}$ was simultaneously recorded on a National Instrument DAQ card (NI USB-6251). Kinetic traces were typically obtained from the average of 64 laser pulses. $2 \mathrm{mg} / \mathrm{mL}$ carbon nitride was dispersed in aqueous solution and transferred to $2 \mathrm{~mm}$ path length cuvettes. Samples were stirred prior to the start of each kinetic acquisition and were measured under argon atmosphere. Data was acquired and processed using home-built software written in the LabVIEW environment.

\section{Photoinduced absorption spectroscopic measurement}

The PIA measurement is based on a home-built pump-probe setup. A $355 \mathrm{~nm}$ LED was used as the pump source to excite the samples with a light on and off time to be $5 \mathrm{~s}$ and $6 \mathrm{~s}$, respectively. The probe light was generated by a 100 W Bentham IL1 quartz tungsten halogen lamp. The light on/off was controlled by National Instrument DAQ card (NI USB-6251). The change of transmitted probe light was monitored at specific wavelength by passing the light through a monochromator (Oriel Cornerstone 130) and detected by a Si photodiode (Hamamatsu S3071). The voltage signal was recorded by the same DAQ board, and the difference in absorbance $(\triangle \mathrm{OD})$ of the sample before and after the LED light pulse is calculated as a function of time.

\section{Photocatalytic experiments and $\mathrm{H}_{2}$ production measurement ${ }^{32}$}

Photocatalytic experiments were carried out by preparing suspensions in borosilicate glass reactors (total volume of 7.74 $\mathrm{mL})$. The reaction solutions $(3 \mathrm{~mL})$ were prepared by mixing exfoliated ${ }^{\mathrm{NCN}} \mathrm{CN}_{\mathrm{x}}$ and $\mathrm{NiP}$ in aqueous potassium phosphate solution $\left(\mathrm{KP}_{\mathrm{i}}\right)$ at $\mathrm{pH} 4.5$ in the presence of 4-methylbenyzl alcohol (4-MBA). The reactors were equipped with a magnetic stirrer, sealed using rubber septa and purged with $\mathrm{N}_{2}$ (containing $2 \% \mathrm{CH}_{4}$ as internal gas chromatography standard). The vials were then placed in water-jacketed reservoir kept at $25{ }^{\circ} \mathrm{C}$ and irradiated using solar light simulator equipped with an air mass 1.5 global filter (AM 1.5G) and water filter to remove infrared irradiation, while stirring. The light simulator was used with and without neural density filters, to provide different light intensities.

The amount of $\mathrm{H}_{2}$ produced in the headspace of the reactors were quantified periodically using a gas chromatography (GC, Agilent 7890A). The headspace gas was injected using an air tight syringe into gas chromatography maintained at $45^{\circ} \mathrm{C}$ while $\mathrm{N}_{2}$ was used as the carrier gas.

\section{Data treatment}


All the data are treated with MATLAB and OriginLab 2017. Data are available upon request to the authors.

\section{References.}

(1) Eberhart, M. S.; Bowers, L. M. R.; Shan, B.; Troian-Gautier, L.; Brennaman, M. K.; Papanikolas, J. M.; Meyer, T. J. Completing a Charge Transport Chain for Artificial Photosynthesis. J. Am. Chem. Soc. 2018, 140 (31), 9823-9826.

(2) Sun, L.; Hammarström, L.; Åkermark, B.; Styring, S. Towards Artificial Photosynthesis: RutheniumManganese Chemistry for Energy Production. Chem. Soc. Rev. 2001, 30 (1), 36-49.

(3) Grätzel, M. Photoelectrochemical Cells. Nature 2001, 414 (6861), 338-344.

(4) Sachs, M.; Pastor, E.; Kafizas, A.; Durrant, J. R. Evaluation of Surface State Mediated Charge Recombination in Anatase and Rutile TiO 2. J. Phys. Chem. Lett. 2016, 7 (19), 3742-3746.

(5) Pesci, F. M.; Cowan, A. J.; Alexander, B. D.; Durrant, J. R.; Klug, D. R. Charge Carrier Dynamics on Mesoporous WO 3 during Water Splitting. J. Phys. Chem. Lett. 2011, 2 (15), 1900-1903.

(6) Zhu, H.; Song, N.; Lian, T. Wave Function Engineering for Ultrafast Charge Separation and Slow Charge Recombination in Type II Core/Shell Quantum Dots. J. Am. Chem. Soc. 2011, 133 (22), 8762-8771.

(7) Zhu, H.; Lian, T. Enhanced Multiple Exciton Dissociation from CdSe Quantum Rods: The Effect of Nanocrystal Shape. J. Am. Chem. Soc. 2012, 134 (27), 11289-11297.

(8) Le Formal, F.; Durrant, J. R.; Mesa, C. A.; Grätzel, M.; Pastor, E.; Pendlebury, S. R.; Tilley, S. D. Rate Law Analysis of Water Oxidation on a Hematite Surface. J. Am. Chem. Soc. 2015, 137 (20), 6629-6637.

(9) Ma, Y.; Mesa, C. A.; Pastor, E.; Kafizas, A.; Francàs, L.; Le Formal, F.; Pendlebury, S. R.; Durrant, J. R. Rate Law Analysis of Water Oxidation and Hole Scavenging on a BiVO 4 Photoanode. ACS Energy Lett. 2016, 1 (3), 618-623.

(10) Grela, M. A.; Colussi, A. J. Kinetics of Stochastic Charge Transfer and Recombination Events in Semiconductor Colloids. Relevance to Photocatalysis Efficiency. J. Phys. Chem. 1996, 100 (46), 1821418221.

(11) Peter, L. M. Energetics and Kinetics of Light-Driven Oxygen Evolution at Semiconductor Electrodes: The Example of Hematite. J. Solid State Electrochem. 2013, 17 (2), 315-326.

(12) Godin, R.; Wang, Y.; Zwijnenburg, M. A.; Tang, J.; Durrant, J. R. Time-Resolved Spectroscopic Investigation of Charge Trapping in Carbon Nitrides Photocatalysts for Hydrogen Generation. J. Am. Chem. Soc. 2017, 139 (14), 5216-5224.

(13) Wu, K.; Chen, Z.; Lv, H.; Zhu, H.; Hill, C. L.; Lian, T. Hole Removal Rate Limits Photodriven H2generation Efficiency in CdS-Pt and CdSe/CdS-Pt Semiconductor Nanorod-Metal Tip Heterostructures. J. Am. Chem. Soc. 2014, 136 (21), 7708-7716.

(14) Cohn, A. W.; Janßen, N.; Mayer, J. M.; Gamelin, D. R. Photocharging ZnO Nanocrystals: Picosecond Hole Capture, Electron Accumulation, and Auger Recombination. J. Phys. Chem. C 2012, 116 (38), 20633-20642.

(15) Romero, N. A.; Nicewicz, D. A. Organic Photoredox Catalysis. Chem. Rev. 2016, 116 (17), 10075-10166.

(16) Marin, M. L.; Santos-Juanes, L.; Arques, A.; Amat, A. M.; Miranda, M. A. Organic Photocatalysts for the Oxidation of Pollutants and Model Compounds. Chem. Rev. 2012, 112 (3), 1710-1750.

(17) Wang, L.; Fernández-Terán, R.; Zhang, L.; Fernandes, D. L. A.; Tian, L.; Chen, H.; Tian, H. Organic Polymer Dots as Photocatalysts for Visible Light-Driven Hydrogen Generation. Angew. Chemie Int. Ed. 2016, 55 (40), 12306-12310.

(18) Wang, X.; Maeda, K.; Thomas, A.; Takanabe, K.; Xin, G.; Carlsson, J. M.; Domen, K.; Antonietti, M. A Metal-Free Polymeric Photocatalyst for Hydrogen Production from Water under Visible Light. Nat. Mater. 2009, 8 (1), 76-80.

(19) Sehnert, J.; Seyfarth, L.; Döblinger, M.; Senker, J.; Schnick, W.; Lotsch, B. V.; Oeckler, O. Unmasking Melon by a Complementary Approach Employing Electron Diffraction, Solid-State NMR Spectroscopy, and 
Theoretical Calculations-Structural Characterization of a Carbon Nitride Polymer. Chem. - A Eur. J. 2007, 13 (17), 4969-4980.

(20) Martin, D. J.; Qiu, K.; Shevlin, S. A.; Handoko, A. D.; Chen, X.; Guo, Z.; Tang, J. Highly Efficient Photocatalytic H 2 Evolution from Water Using Visible Light and Structure-Controlled Graphitic Carbon Nitride. Angew. Chemie Int. Ed. 2014, 53 (35), 9240-9245.

(21) Ou, H.; Lin, L.; Zheng, Y.; Yang, P.; Fang, Y.; Wang, X. Tri- s -Triazine-Based Crystalline Carbon Nitride Nanosheets for an Improved Hydrogen Evolution. Adv. Mater. 2017, 29 (22), 1700008.

(22) Lotsch, B. V.; Döblinger, M.; Sehnert, J.; Seyfarth, L.; Senker, J.; Oeckler, O.; Schnick, W. Unmasking Melon by a Complementary Approach Employing Electron Diffraction, Solid-State NMR Spectroscopy, and Theoretical Calculations-Structural Characterization of a Carbon Nitride Polymer. Chem. - A Eur. J. 2007, 13 (17), 4969-4980.

(23) Lin, J.; Pan, Z.; Wang, X. Photochemical Reduction of CO 2 by Graphitic Carbon Nitride Polymers. ACS Sustain. Chem. Eng. 2014, 2 (3), 353-358.

(24) Qin, J.; Wang, S.; Ren, H.; Hou, Y.; Wang, X. Photocatalytic Reduction of CO 2 by Graphitic Carbon Nitride Polymers Derived from Urea and Barbituric Acid. Appl. Catal. B Environ. 2015, 179, 1-8.

(25) Ong, W.-J.; Tan, L.-L.; Ng, Y. H.; Yong, S.-T.; Chai, S.-P. Graphitic Carbon Nitride (g-C 3 N 4 )-Based Photocatalysts for Artificial Photosynthesis and Environmental Remediation: Are We a Step Closer To Achieving Sustainability? Chem. Rev. 2016, 116 (12), 7159-7329.

(26) Corp, K. L.; Schlenker, C. W. Ultrafast Spectroscopy Reveals Electron-Transfer Cascade That Improves Hydrogen Evolution with Carbon Nitride Photocatalysts. J. Am. Chem. Soc. 2017, 139 (23), 7904-7912.

(27) Merschjann, C.; Tschierlei, S.; Tyborski, T.; Kailasam, K.; Orthmann, S.; Hollmann, D.; Schedel-Niedrig, T.; Thomas, A.; Lochbrunner, S. Complementing Graphenes: 1D Interplanar Charge Transport in Polymeric Graphitic Carbon Nitrides. Adv. Mater. 2015, 27 (48), 7993-7999.

(28) Shi, X.; Fujitsuka, M.; Lou, Z; Zhang, P.; Majima, T. In Situ Nitrogen-Doped Hollow-TiO2/g-C3N4 Composite Photocatalysts with Efficient Charge Separation Boosting Water Reduction under Visible Light. $J$. Mater. Chem. A 2017, 5 (20), 9671-9681.

(29) Elbanna, O.; Fujitsuka, M.; Majima, T. G-C3N4/TiO2 Mesocrystals Composite for H2 Evolution under Visible-Light Irradiation and Its Charge Carrier Dynamics. ACS Appl. Mater. Interfaces 2017, 9 (40), 3484434854.

(30) Rabe, E. J.; Corp, K. L.; Sobolewski, A. L.; Domcke, W.; Schlenker, C. W. Proton-Coupled Electron Transfer from Water to a Model Heptazine-Based Molecular Photocatalyst. J. Phys. Chem. Lett. 2018, 9 (21), 62576261.

(31) Lau, V. W.; Moudrakovski, I.; Botari, T.; Weinberger, S.; Mesch, M. B.; Duppel, V.; Senker, J.; Blum, V.; Lotsch, B. V. Rational Design of Carbon Nitride Photocatalysts by Identification of Cyanamide Defects as Catalytically Relevant Sites. Nat. Commun. 2016, 7, 12165.

(32) Kasap, H.; Caputo, C. A.; Martindale, B. C. M.; Godin, R.; Lau, V. W. H.; Lotsch, B. V.; Durrant, J. R.; Reisner, E. Solar-Driven Reduction of Aqueous Protons Coupled to Selective Alcohol Oxidation with a Carbon Nitride-Molecular Ni Catalyst System. J. Am. Chem. Soc. 2016, 138 (29), 9183-9192.

(33) Lau, V. W.; Klose, D.; Kasap, H.; Podjaski, F.; Pignié, M.-C.; Reisner, E.; Jeschke, G.; Lotsch, B. V. Dark Photocatalysis: Storage of Solar Energy in Carbon Nitride for Time-Delayed Hydrogen Generation. Angew. Chemie Int. Ed. 2017, 56 (2), 510-514.

(34) Podjaski, F.; Kröger, J.; Lotsch, B. V. Toward an Aqueous Solar Battery: Direct Electrochemical Storage of Solar Energy in Carbon Nitrides. Adv. Mater. 2018, 30 (9), 1705477.

(35) Yang, S.; Gong, Y.; Zhang, J.; Zhan, L.; Ma, L.; Fang, Z.; Vajtai, R.; Wang, X.; Ajayan, P. M. Exfoliated Graphitic Carbon Nitride Nanosheets as Efficient Catalysts for Hydrogen Evolution under Visible Light. Adv. Mater. 2013, 25 (17), 2452-2456.

(36) Kasap, H.; Achilleos, D. S.; Huang, A.; Reisner, E. Photoreforming of Lignocellulose into $\mathrm{H}_{2}$ Using Nanoengineered Carbon Nitride under Benign Conditions. J. Am. Chem. Soc. 2018, 140 (37), 11604-11607. 
(37) Yang, S.; Gong, Y.; Zhang, J.; Zhan, L.; Ma, L.; Fang, Z.; Vajtai, R.; Wang, X.; Ajayan, P. M. Exfoliated Graphitic Carbon Nitride Nanosheets as Efficient Catalysts for Hydrogen Evolution Under Visible Light. Adv. Mater. 2013, 25 (17), 2452-2456.

(38) Lyth, S. M.; Nabae, Y.; Moriya, S.; Kuroki, S.; Kakimoto, M.; Ozaki, J.; Miyata, S. Carbon Nitride as a Nonprecious Catalyst for Electrochemical Oxygen Reduction. J. Phys. Chem. C 2009, 113 (47), 20148-20151.

(39) Zheng, Y.; Jiao, Y.; Chen, J.; Liu, J.; Liang, J.; Du, A.; Zhang, W.; Zhu, Z.; Smith, S. C.; Jaroniec, M.;Lu, G. Qiao, S. Nanoporous Graphitic-C 3 N 4 @ Carbon Metal-Free Electrocatalysts for Highly Efficient Oxygen Reduction. J. Am. Chem. Soc. 2011, 133 (50), 20116-20119.

(40) Yang, S.; Feng, X.; Wang, X.; Müllen, K. Graphene-Based Carbon Nitride Nanosheets as Efficient Metal-Free Electrocatalysts for Oxygen Reduction Reactions. Angew. Chemie 2011, 123 (23), 5451-5455.

(41) Khan, M. A.; Maity, P.; Al-Oufi, M.; Al-Howaish, I. K.; Idriss, H. Electron Transfer of the Metal/Semiconductor System in Photocatalysis. J. Phys. Chem. C 2018, 122 (29), 16779-16787.

(42) Noda, Y.; Merschjann, C.; Tarábek, J.; Amsalem, P.; Koch, N.; Bojdys, M. J. Directional Charge Transport in Layered, Two-Dimensional Triazine-Based Graphitic Carbon Nitride. Angew. Chemie Int. Ed. 2019.

(43) Nelson, J.; Chandler, R. E. Random Walk Models of Charge Transfer and Transport in Dye Sensitized Systems. Coord. Chem. Rev. 2004, 248 (13-14), 1181-1194.

(44) Nelson, J. Continuous-Time Random-Walk Model of Electron Transport in Nanocrystalline TiO2 Electrodes. Physical Review B. 1999, pp 15374-15380.

(45) Tachiya, M.; Seki, K. Theory of Bulk Electron-Hole Recombination in a Medium with Energetic Disorder. Phys. Rev. B 2010, 82 (8), 085201.

(46) Walsh, J. J.; Jiang, C.; Tang, J.; Cowan, A. J. Photochemical CO2 Reduction Using Structurally Controlled GC3N4. Phys. Chem. Chem. Phys. 2016, 18 (36), 24825-24829.

(47) The extinction coefficient of electrons in $\mathrm{CNx}$ is unknown in literatures. Here we use the widely accepted value from electrons in $\mathrm{TiO}_{2}$ as an approximation to numerically calculate the densities of charges in the current yystems. The corrected extinction will change the absolute values obtained here, but not change the order difference for the accumulated and photogenerated electrons and therefore the general conclusions of the paper.

(48) Durrant, J. R.; Haque, S. A.; Palomares, E. Towards Optimisation of Electron Transfer Processes in Dye Sensitised Solar Cells. Coord. Chem. Rev. 2004, 248 (13-14), 1247-1257.

(49) Gross, M. A.; Reynal, A.; Durrant, J. R.; Reisner, E. Versatile Photocatalytic Systems for H2 Generation in Water Based on an Efficient DuBois-Type Nickel Catalyst. J. Am. Chem. Soc. 2014, 136 (1), 356-366.

(50) Kasap, H.; Godin, R.; Jeay-Bizot, C.; Achilleos, D. S.; Fang, X.; Durrant, J. R.; Reisner, E. Interfacial Engineering of a Carbon Nitride-Graphene Oxide-Molecular Ni Catalyst Hybrid for Enhanced Photocatalytic Activity. ACS Catal. 2018, 8 (8), 6914-6926.

(51) Chica, B.; Wu, C. H.; Liu, Y.; Adams, M. W. W.; Lian, T.; Dyer, R. B. Balancing Electron Transfer Rate and Driving Force for Efficient Photocatalytic Hydrogen Production in CdSe/CdS Nanorod-[NiFe] Hydrogenase Assemblies. Energy Environ. Sci. 2017, 10 (10), 2245-2255.

(52) Zhu, H.; Song, N.; Lv, H.; Hill, C. L.; Lian, T. Near Unity Quantum Yield of Light-Driven Redox Mediator Reduction and Efficient H 2 Generation Using Colloidal Nanorod Heterostructures. J. Am. Chem. Soc. 2012, 134 (28), 11701-11708.

(53) Weiss, E. A. Designing the Surfaces of Semiconductor Quantum Dots for Colloidal Photocatalysis. ACS Energy Lett. 2017, 2 (5), 1005-1013.

(54) Morris-Cohen, A. J.; Peterson, M. D.; Frederick, M. T.; Kamm, J. M.; Weiss, E. A. Evidence for a ThroughSpace Pathway for Electron Transfer from Quantum Dots to Carboxylate-Functionalized Viologens. J. Phys. Chem. Lett. 2012, 3 (19), 2840-2844.

(55) Martindale, B. C. M.; Hutton, G. A. M.; Caputo, C. A.; Reisner, E. Solar Hydrogen Production Using Carbon Quantum Dots and a Molecular Nickel Catalyst. J. Am. Chem. Soc. 2015, 137 (18), 6018-6025. 
(56) This aggregation is suspected to be due to the change of surface charge of ${ }^{\mathrm{NCN}} \mathrm{CNx}$ due to the surface adsorption of $\mathrm{MV}^{2+}$ onto the negative charge ${ }^{\mathrm{NCN}} \mathrm{CNx}$ surface, which was previously widely shown in many $\mathrm{MV}^{2+}$ and quantum dots systems.

(57) Le Formal, F.; Pendlebury, S. R.; Cornuz, M.; Tilley, S. D.; Grätzel, M.; Durrant, J. R. Back Electron-Hole Recombination in Hematite Photoanodes for Water Splitting. J. Am. Chem. Soc. 2014, 136 (6), 2564-2574.

(58) Rinehart, J. D.; Schimpf, A. M.; Weaver, A. L.; Cohn, A. W.; Gamelin, D. R. Photochemical Electronic Doping of Colloidal CdSe Nanocrystals. J. Am. Chem. Soc. 2013, 135 (50), 18782-18785. 
For Table of Contents Only:
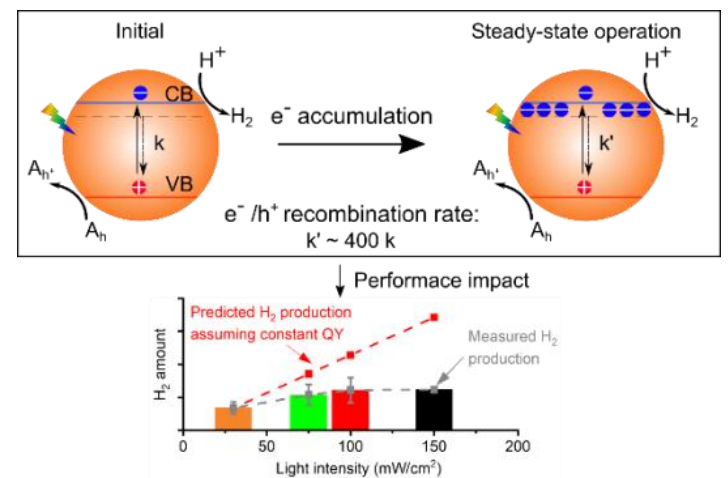